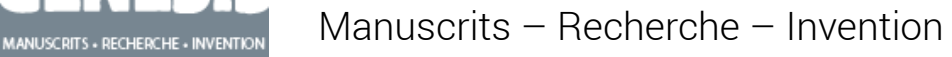

47 | 2018

Entrer en listes

\title{
Jean-Philippe Toussaint - Débris et drapés
}

\section{Gaspard Turin}

\section{OpenEdition}

Journals

Édition électronique

URL : http://journals.openedition.org/genesis/3691

DOI : 10.4000/genesis.3691

ISSN : 2268-1590

\section{Éditeur :}

Presses universitaires de Paris Sorbonne (PUPS), Société internationale de génétique artistique littéraire et scientifique (SIGALES)

\section{Édition imprimée}

Date de publication : 15 décembre 2018

Pagination : 107-112

ISBN : 979-10-231-06282

ISSN : 1167-5101

\section{Référence électronique}

Gaspard Turin, « Jean-Philippe Toussaint - Débris et drapés », Genesis [En ligne], 47 | 2018, mis en ligne le 15 décembre 2019, consulté le 21 janvier 2021. URL : http://journals.openedition.org/genesis/3691 ; DOI : https://doi.org/10.4000/genesis.3691 


\title{
Débris et drapés Entretien avec Jean-Philippe Toussaint
}

\author{
Propos recueillis par Gaspard Turin
}

\begin{abstract}
L'entretien transcrit ci-dessous porte essentiellement sur les documents génétiques relatifs au cycle dit «de Marie» publié aux Éditions de Minuit entre 2002 et 2013. Un grand nombre de ces documents sont consultables sur le site internet de l'auteur ${ }^{1}$, sous différentes formes et reflétant différents états de la genèse de ces quatre romans.
\end{abstract}

\section{De la réduction à l'expansion}

Gaspard Turin - J'aimerais que nous évoquions tout d'abord la situation générale des dossiers de préparation mis en ligne sur ton site. Il semblerait que, du premier (Faire l'amour) au dernier (Nue) des quatre romans du cycle de Marie, le volume de documents génétiques ait sensiblement augmenté. Est-ce que la complexité des brouillons s'est effectivement développée à tes yeux au fil du cycle?

Jean-Philippe Toussaint - Très franchement, je n'y avais jamais réfléchi. Je suis prêt à admettre qu'il y ait moins de matériel pour Faire l'amour et de plus en plus ensuite, oui. J'ai un souvenir assez précis de la genèse de ce roman, où j'ai imaginé des scènes qui ont complètement disparu. Pas même des scènes, des hypothèses, de simples idées. Mais en fait, assez vite, je me suis rendu compte que ces premières idées auraient mené à quelque chose de trop ample. Je n'ai rien gardé, rien rédigé de ces idées parce que je me serais noyé dans un abus de références. Par intuition, je vais toujours vers le resserrement.

GT - Pourtant, rétrospectivement, Faire l'amour n'est pas un roman autonome; il s'y trouve de très nombreux indices qui ouvrent le cycle et trouvent un écho dans les romans suivants...

$J P T$ - Bien sûr. Dès l'avant-texte de Faire l'amour, il était prévu que je traite l'enterrement du père de Marie - un épisode qui n'apparaîtra que dans Fuir. Lorsqu'on relit attentivement le premier roman, on s'aperçoit très vite, dans les dix premières pages, que Marie pleure parce que son père est mort. Je savais que son père serait enterré à l'île d'Elbe, où il avait une propriété; je ne savais pas encore à quoi ressemblerait vraiment la scène, mais cet enterrement était prévu. Cet épisode est l'une des raisons qui m'ont amené à faire un cycle, à écrire une suite, ce qui n'était pas prévu au moment de la rédaction de Faire l'amour. Parce que je n'avais pas envisagé de suite, je ne travaillais les avant-textes que d'un seul roman. Il s'est trouvé par la suite que certains éléments qu'ils contenaient n'avaient pas été utilisés, lesquels m'ont amené à comprendre que j'étais en train de construire un ouvrage de plus grande ampleur. En commençant Fuir, j'ai assez vite saisi que cette façon d'écrire un nouveau livre, mais qui ferait partie d'un ensemble, pourrait se poursuivre. Ayant appris à travailler dans cette temporalité à la fois courte et longue, j'ai logiquement amassé plus de matière. Mon instinct de resserrement s'est heurté à la logique exponentielle de ce projet littéraire particulier. En écrivant Fuir, je devais à la fois nourrir le projet à court terme qu'était Fuir et le projet à long terme du cycle, de cette poursuite de l'écriture dont j'avais l'idée à ce moment.

GT - Trouverait-on encore aujourd'hui, dans les brouillons, des éléments qui n'ont pas abouti ou qui n'ont pas été exploités?

$J P T$ - Il y en a énormément, de ces choses qui apparaissent dans les avant-textes mais qui n'ont pas été utilisées dans le texte final. Il serait intéressant d'étudier ces scories, ces débris. Pour préparer l'entretien, je suis retourné jeter un

1. www.jptoussaint.com. 
coup d'œil aux manuscrits. Et j'ai trouvé quelque chose qui m'a enchanté, en prévision de la publication de M.M.M.M. ${ }^{2}$. Dans les notes de Fuir, j' ai lu la note suivante : «aime, aime, aime, aime», comme une prière, une litanie ou une invocation à aimer Marie. Grâce à notre entretien, j'ai retrouvé un passage clé de ce projet!

\section{Montage / tournage / scénario}

GT - Concernant l'architecture du site et l'organisation des documents préparatoires qu'on y rencontre, on observe trois rubriques générales : "États du manuscrit», "Plans, variantes, débris » et «Brouillons, notes, dessins». Pourquoi cette répartition? À quoi correspond-elle?

$J P T$ - Il y a une différence très nette entre les deux premières rubriques, qui contiennent uniquement des documents issus de pages écrites à l'ordinateur, et la troisième, qui est constituée de scans. Les deux premières proviennent de documents numériques qui ne sont pas séparés dans mon ordinateur. Cette séparation n'existe que pour les besoins de la publication sur Internet. Quand je travaille, je ne sépare jamais les «états du manuscrit» des «plans, variantes, débris ». Mon document de travail se présente sous la forme de l'état du manuscrit - c'est le moment où j'en suis dans l'écriture. Ensuite, il m'arrive d'avoir envie de faire un état des lieux : je fais donc un plan (il est assez rare que je fasse un plan à court terme, c'est plutôt un plan à plus long terme, pour une deuxième partie ou pour un livre à venir). Ce plan se trouve alors plus loin dans le document. Et tout à la fin, il y a ce que j'appelle «débris » et qui est un mot que j'aime beaucoup, auquel j'ai vraiment réfléchi. J'aimerais un jour publier un texte qui s'intitulerait ainsi, un peu dans l'idée des Fusées de Baudelaire. J'aime ce mot aussi pour ce qu'il peut avoir de péjoratif : il s'agirait dès lors de le revendiquer et de le valoriser. Ces débris se trouvent toujours à la toute fin du document, ce qui me permet de m'y retrouver. Je reprends la rédaction là où je l'ai laissée, à la fin de l' «état du manuscrit»; je regarde où j'en suis. Si je constate à propos de tel ou tel élément du texte que, finalement, je ne m'en sers pas, je fais un couper-coller et je le place à la fin du document.
GT - Les «états du manuscrit» apparaissent dès lors comme épurés, nettoyés de leurs scories. S'en tenir aux versions successives de ces états pourrait donner l'impression d'une progression continue, d'une session d'écriture à l'autre, avec à chaque nouvelle étape une récupération impeccable du fil narratif de la précédente...

$J P T-\ldots$ Ce qui n'est donc pas tout à fait le cas. Cette séparation artificielle présente l'avantage de clarifier les choses, à mon avis. Pour moi, je m'en sors, mais il me semble qu'un lecteur extérieur ne pourrait pas s'y retrouver si les plans et les débris étaient restés intégrés aux «états du manuscrit »... Et en fait, ce désir de clarté correspond quand même à une réalité, légèrement tronquée, il est vrai, dans ce souci de présentation. Dans mon esprit, quand j'ouvre mon document et que je travaille, il me faut cette clarté, cette propreté, pour pouvoir progresser de manière fluide. Mais ce que ta question met en évidence surtout, c'est qu'il n'y a pas de séparation précise entre le moment de la rédaction et un avant-texte qui en serait séparé. Je ne commence pas à écrire avec l'aide d'un avant-texte dont je disposerais sans avoir encore rien rédigé. Je travaille en même temps sur quelque chose de déjà rédigé, qui est généralement le début, et parallèlement sur l'avant-texte de la suite. En fait, il n'y a pratiquement pas d'avant-texte qui concerne le début - puisque, dès que je commence, je commence au début, dans l'ordre, et en même temps je produis de l'avant-texte pour la suite.

\section{GT - Comme deux machines qui fonctionnent en parallèle.}

$J P T$ - Oui. Pour prendre une métaphore cinématographique, je tourne, je monte, et en même temps j'écris le scénario en fonction de ce que je tourne et suis en train de monter. Le scénario n'est pas écrit à l'avance : c'est parce que je suis en train de tourner quelque chose que je vais écrire le scénario de la suite. Il est très rare en fait que j'aie un plan du livre indépendamment du processus d'écriture en cours.

2. Le cycle a été récemment republié en un volume unique : M.M.M.M., Paris, Minuit, 2017. 


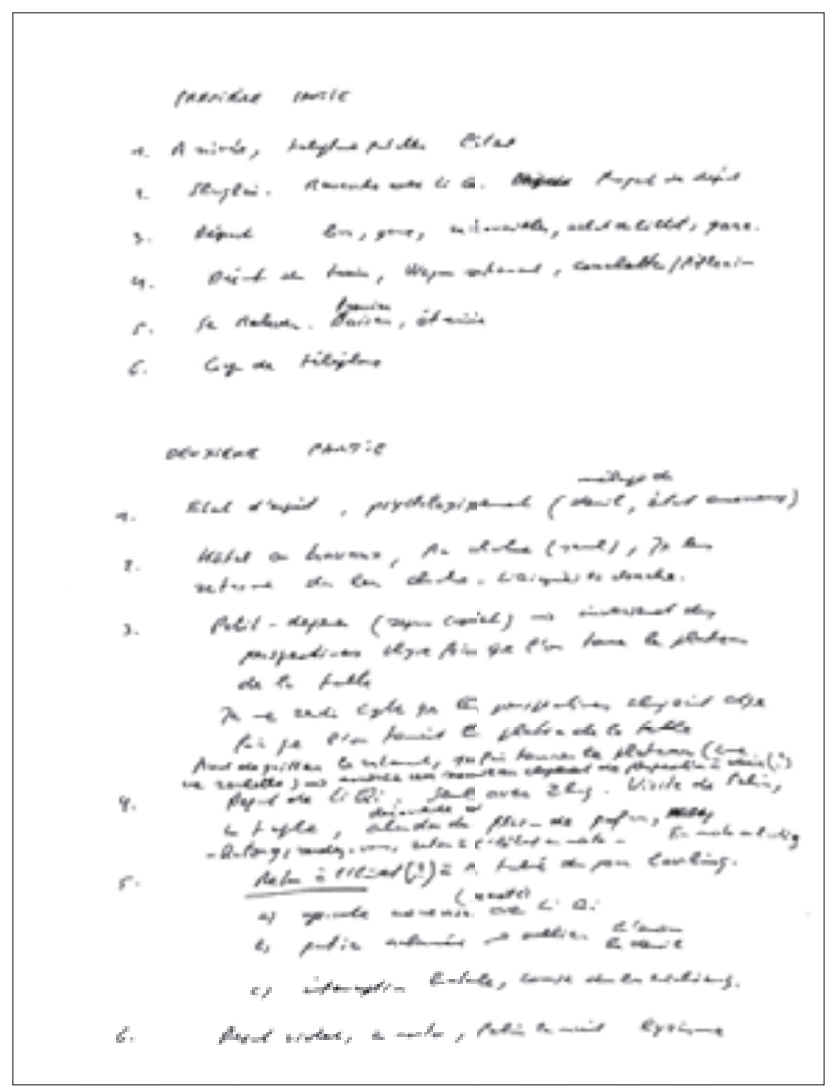

Fig. 1 : Brouillon de Fuir.

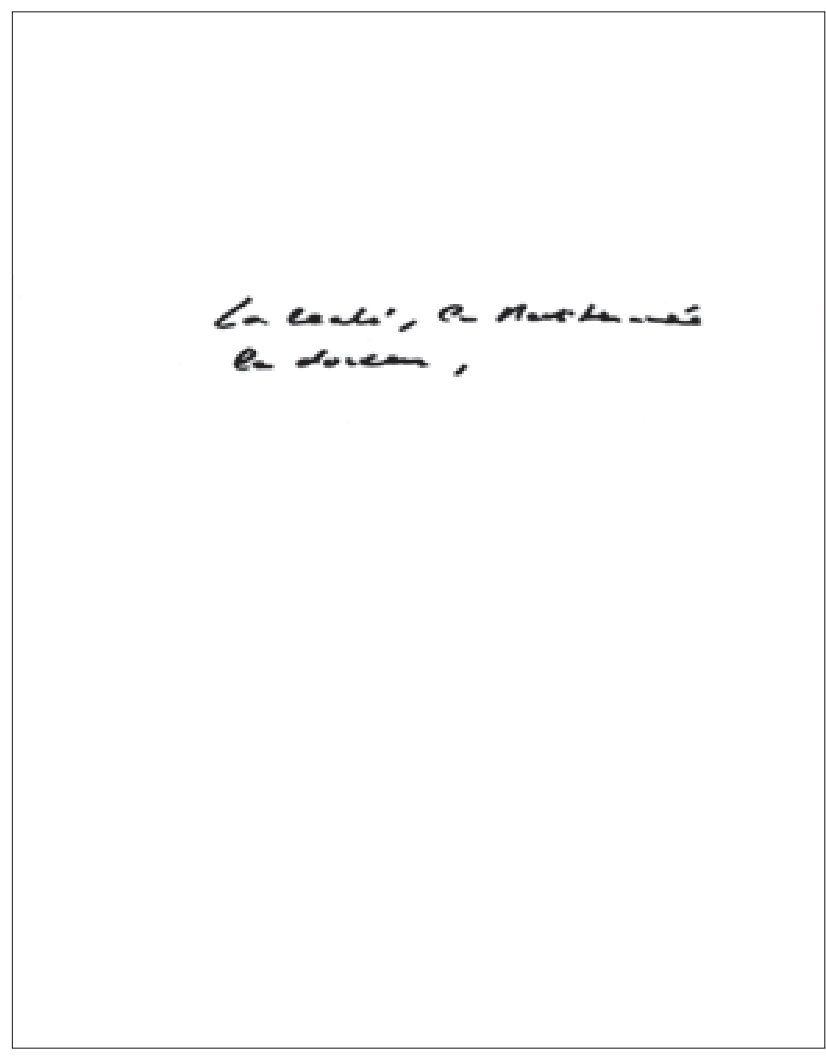

Fig. 2 : «Notes manuscrites [troisième partie]» de La Vérité sur Marie. Trois mots occupent à eux seuls une page sinon vide.

GT - Est-ce que tout le livre est là, sur la page?

$J P T$ - Pas tout à fait, puisqu'il manque la troisième partie. Pour la deuxième, il y a plus de détails, parce que je rappelle les points que je vais devoir traiter. Pour la première, je résume ce que j'ai déjà fait, il y a moins de détails. Quant à savoir pourquoi avoir fait ce plan rétrospectif, je me comparerais à un musicien qui fait des gammes avant de se remettre au travail. Et puis c'est assez joli, non? Il y a aussi un sentiment graphique à l'œuvre, qui participe d'une dimension très importante pour moi, y compris dans le roman publié, où je ne vais jamais à la ligne (il y a toujours une ligne de blanc entre deux paragraphes). Même sans entrer dans la lecture, on voit qu'il y a des rectangles de différentes tailles. Ce souci esthétique, je le retrouve dans ma manière d'écrire mes notes à la main. Ce passage, par exemple, «La beauté, la Méditerranée / la douceur» (fig. 2) - c'est assez beau visuellement. C'est presque de la calligraphie.

$\mathrm{GT}-C$ 'est d'ailleurs, on y vient, une liste. La qualité esthétique dont tu parles doit-elle quelque chose à l'effet est postérieure et correspond aux deux sessions de travail de 2003, en particulier mai-juin 2003 où j’ai terminé le livre. 
de juxtaposition? D'autre part, s'agit-il d'une liste programmatique de l'écriture?

$J P T$ - Oui et oui. Je conçois même avoir fait preuve d'une certaine coquetterie dans la confection de cette note - que je considère aussi bien comme une calligraphie qu'un poème et qu'une consigne. Car il s'agit bien d'une sorte de programme, ou plutôt d'un but à atteindre. Je fais souvent cela : j'écris en majuscules ALLER DE L'AVANT - qui est une injonction à moi-même, une façon de me dire d'arrêter de procrastiner, de ne pas rester bloqué sur un paragraphe. Je me donne souvent cette consigne, parce que j'ai tendance à relire, à reprendre. Comme je l'ai écrit dans L'Urgence et la Patience, il faut des moments d'attente pour que les choses puissent survenir ${ }^{3}$. Aller de l'avant - je me le conseille, mais il ne suffit pas de l'écrire pour que cela fasse effet. Je ne peux pas aller de l'avant au troisième jour de l'écriture. Ça viendra au bout de, mettons, dix jours. Il faut que, durant sept ou huit jours, j'aie perdu du temps à des tâches a priori inutiles : relire, reprendre -, que j'aie accumulé suffisamment d'énergie pour aller de l'avant.

GT - La qualité esthétique de cette liste, et plus encore de celles qui composent les plans des première et deuxième parties de Fuir, me semble identique à celle de ta prose. L'adjectif qui lui correspond le mieux est peut-être "fluide»: il y a une qualité liquide qui impose à ta syntaxe une souplesse, laquelle ne se satisfait pas de la fragmentation généralement associée à la forme liste.

$J P T$ - Effectivement, je vise la fluidité en général.

$\mathrm{GT}$ - Le fameux pronom «qui » ou «que», ouvrant une relative au-delà des limites ordinaires de la phrase (comme dans "L'air du matin était frais, qu'elle sentait vivifiant contre son visage ${ }^{»)}$ ) participe aussi de cette fluidité, liée à une nécessaire vitesse de lecture...

$J P T$ - C'était surtout dans La Réticence que j'ai fait usage de ce «qui » postposé, on me l'a beaucoup fait remarquer ${ }^{5}$, je le fais moins maintenant, je m'en méfie... On trouve cette tournure chez Gide. Je la réutilise de temps en temps, mais il faut vraiment que ça se justifie. Ça ne fonctionne que si l'écriture a un élan, et que dans cet élan, la forme de cette relative s'applique. À lire lentement, on pourrait penser que la relative s'applique autrement, au complément de nom par exemple. Si on lit «La fille de ma sœur, qui m'accompagnait», on comprend bien que le pronom se rapporte à ma nièce, pas à ma sœur - mais c'est l'élan de la lecture qui permet de désambiguïser cette question. Et cet élan donc, exige effectivement une certaine vitesse de lecture qui ne peut s'accommoder de l'esthétique fragmentaire.

GT - Tu parles de Proust comme d'un modèle6. Doit-on observer une parenté entre ton écriture et la tendance proustienne à enrichir l'objet du souvenir d'une saturation de la sensation? À composer le texte comme un continuum fusionnant l'expérience fragmentaire en un tout lié, fluidifié?

$J P T-\ldots$ Oui et non. Le contraire, le fragment m'intéresse aussi. On m'avait demandé pourquoi j'avais numéroté les paragraphes de La Salle de bain. J'avais répondu que je voulais casser la fluidité de la lecture. Rappeler qu'on était dans un livre. Numéroter les paragraphes participait d'une logique de fragmentation. Même si, en même temps, je travaillais les transitions pour chercher la fluidité. Je cherchais un toboggan qui pourrait induire la liquidité de la lecture, tout en construisant des espaliers pour l'arrêter.

GT - Mais ne parles-tu pas ici de la première partie de ton ouvre? Il me semble que cette tendance disparaît par la suite...

$J P T-$ C'est vrai que je recherche beaucoup plus la fluidité aujourd'hui. Mais ça n'est pas pour contrer le fragment à tout prix. Il m'intéresse aussi de laisser d'énormes blancs et d'énormes territoires non traités; je n'ai pas envie de tout remplir. Il y a des îles. Le cycle de Marie dure environ sept ans, dont le récit comporte beaucoup d'ellipses. J'ai construit cela de manière très consciente. Mais une fois que je m'attelle à une scène, en effet, je veux la saturer, et cette idée de saturation est très présente chez Proust. À un degré parfois

3. L'Urgence et la Patience, Paris, Minuit, 2012, notamment p. 28.

4. La Vérité sur Marie, Paris, Minuit, 2009, p. 155.

5. En particulier Pierre Jourde dans son pamphlet La Littérature sans estomac, Paris, L'Esprit des péninsules, coll. «Pocket», 2002, p. 199-204. 6. L'Urgence et la Patience, op. cit., p. 61-68. 
incroyable : j'ai relu l'année dernière Sodome et Gomorrhe, et au moment où le narrateur rend visite aux Verdurin, il y a le personnage de Brichot, qui connaît l'étymologie de tous les noms de lieux. De manière absolument invraisemblable, ces explications étymologiques se prolongent sur cinq ou six pages. Alors même que Brichot a disparu, le narrateur, infesté, continue sur sa lancée, sans pouvoir s'en empêcher. L'autre exemple, c'est le patron de l'hôtel de Balbec qui, un peu comme Coluche, emploie un mot pour un autre. Cela finit par n'être plus drôle, parce qu'il y en a trop. Il en fait trop. On quitte le vraisemblable, dans cette saturation. C'est un exemple un peu extrême, quelque chose que je m'applique à ne pas faire. Mais c'est aussi un danger que je cours, croyant bien faire. Dans ces cas-là, je mets la barre très haut, parce que c'est Proust.

GT - L'exemple de Brichot est fascinant, parce qu'il fait écho au passage de rêverie du narrateur sur les noms des stations sur l'horaire du train. Les noms constituent justement une liste, à l'aspect fragmenté, mais le propos de ce passage revient précisément à dé-fragmenter le réel pour lui donner le liant que les sensations et la mémoire du narrateur se chargent de reconstruire.

$J P T$ - En effet, le trajet du train est rythmé par les arrêts et dans le récit par les noms de la liste.

\section{Listage, lissage}

GT - Pratiques-tu la liste dans la préparation de tes romans?

$J P T$ - Comme je te le disais lors de nos premiers échanges, je n'ai pas l'impression d'en faire. Je fais des plans, mais pour moi il ne s'agit pas de listes. C'est peut-être d'une liste trop pure dont je me fais l'idée, quelque chose de perecquien... Il doit y avoir d'autres exemples d'auteurs qui cherchent presque à épuiser les synonymes, ou qui ont le goût d'accumuler des éléments semblables généralement très voisins. C'est un procédé que je n'utilise que très rarement et, si je le fais, j'ai tendance à me corriger et à ne pas le garder. Il n'est pas exclu que j'aie déjà utilisé des listes, mais si je les vois je les corrige, parce qu'esthétiquement ça ne va pas dans mes romans.

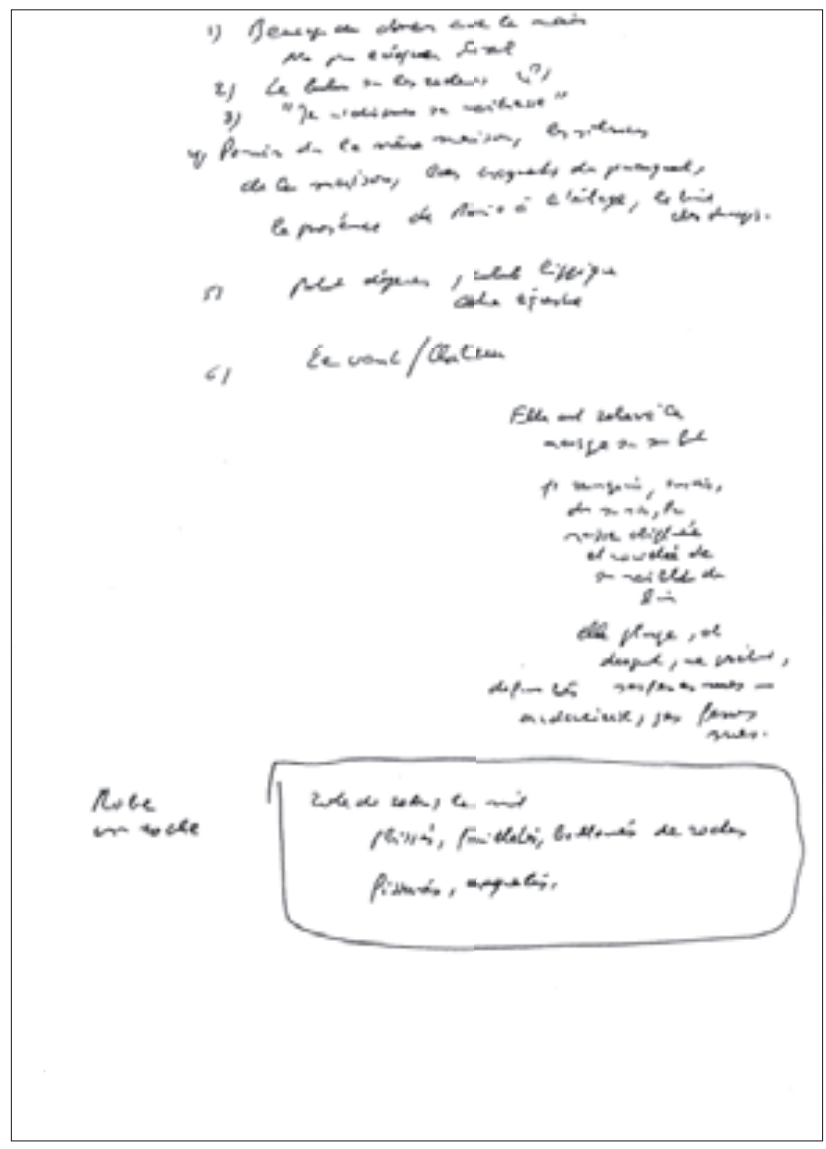

Fig. 3 : Quatrième page du dossier de La Vérité sur Marie, «Notes manuscrites (troisième partie)».

GT - J'aimerais prendre l'exemple de cette page (fig. 3), la quatrième du document tirée du dossier de La Vérité sur Marie, «Notes manuscrites (troisième partie)». Tu serais d'accord qu'il y a ici quelque chose qui ressemble à une liste? Et même à une liste dans la liste, si je prends par exemple le point 4) : "Dormir dans la même maison, les silences de la maison les craque[men]ts du parquet, la présence de Marie à l'étage, le bruit des draps. » Ou encore la «robe en roche» et sa liste d'adjectifs, au bas de la même page. On pourrait dire qu'il y a ici, à l'œuvre, une logique de liste dans la construction du plan?

$J P T$ - Je comprends bien comment tu l'entends; oui, il y a une exposition, je vais à la ligne... effectivement, c'est indéniable. Mais les éléments des brouillons manuscrits sont d'une autre nature que la liste telle que je me la représente. C'est qu'elle a, ici, une fonction utilitaire et non esthétique. En réalité, l'utilisation esthétique de la liste ne me correspond pas, mais son côté pratique, sa capacité à faire plan, me plaît beaucoup parce que j'ai un esprit très cartésien et logique 
et j'adore numéroter. J'aime les 1), les a., j'aime beaucoup les plans.

GT - Si je consulte le roman publié, j'observe que les éléments figurant la rubrique 4) se retrouvent presque à l'identique, sous la forme des syntagmes qu'ils constituaient dans le plan, mais dans le cadre d'un développement narratif et descriptif qui court sur une quinzaine de pages et dans un ordre différent - ce qui en fait une liste 7 . Il ne manque que «le bruit des draps», que je n'ai pas retrouvé tel quel.

$J P T$ - Oui, c'est intéressant de voir ce que ces éléments sont devenus. Ils font figure de matière de départ, qu'ensuite je malaxe et transforme. Et «le bruit des draps», ça n'est pas extraordinaire. Je me suis dit que je pouvais trouver mieux, je ne l'ai pas gardé tel quel, j'ai dû le transformer en autre chose ${ }^{8}$.

GT - Ces éléments font liste dans les brouillons, mais même si on les retrouve parfois sous la même forme syntagmatique dans le texte final, tu ne les as pas gardés dans l'ordre dans lequel ils se trouvent dans le manuscrit; ça se diffuse, ça se lisse dans la page. La liste dans le manuscrit apparaît sans hiérarchie entre les éléments, qui semblent destinés à n'être que juxtaposés, en vue d'une description qui va faire sens en soi mais dont le vecteur directionnel, le telos, n'est pas donné par cette liste initiale. En somme, la rédaction lutte contre la liste.
$J P T$ - Oui. Il y a d'ailleurs, j'y pense maintenant, un passage plein d'adjectifs de couleur dans La Vérité sur Marie ${ }^{9}$. On peut observer tout un travail de mise en forme, de construction de la description. Même dans la mesure où il devait y avoir une liste à la base de ces pages (j'avais accumulé beaucoup de documentation, j'avais tout un nuancier de couleurs), le résultat final, très travaillé, s'éloigne autant qu'il se peut d'une simple accumulation d'adjectifs. Il y a souvent une tension, dans mon écriture, entre une tendance minimaliste et un aspect quasi baroque, qui survient, par exemple, quand il est question de mode. La tentation du minimalisme subsiste, mais quand je parle de mode, je n'ai pas peur de convoquer des adjectifs. Mais c'est sans doute un autre sujet...

7. La Vérité sur Marie, op. cit. : «dormir dans la même maison» p. 171, «les silences de la maison»p. 184, «les craquements du parquet»p. 183, «la présence de Marie à l'étage» p. 182.

8. «Les violentes tempêtes de draps», ibid., p. 183.

9. Ibid., p. 140. 